\title{
Monte Carlo simulations of Upsilon meson production
}

\section{Jaroslav Bielcik, Jakub Ceska, ${ }^{*}$ Leszek Kosarzewski and Miroslav Myska}

Faculty of Nuclear Sciences and Physical Engineering, Czech Technical University in Prague, Brehova 7, 11519 Praha 1, Czech Republic

E-mail: ceskajak@fjfi.cvut.cz

$\Upsilon$ meson production dependence on charged particle multiplicity involves the interplay between soft and hard QCD processes. Normalised multiplicity dependence is a meaningful tool for understanding the particle production mechanisms. The observed strong increase may be explained by multi-parton interactions, string percolation or color glass condensate saturation effects. This paper presents Monte Carlo simulations of $\Upsilon$ meson production in proton-proton collisions at $\sqrt{s}=500 \mathrm{GeV}$ with PYTHIA and Herwig generators. The aim of the simulations is to explore the dependence of normalised $\Upsilon$ meson yield on normalised event multiplicity. Herwig with $k_{\perp}>4 \mathrm{GeV} / \mathrm{c}$ shows a linear dependence of $N_{\Upsilon} /\left\langle N_{\Upsilon}\right\rangle$ vs. $N_{\mathrm{ch}} /\left\langle N_{\mathrm{ch}}\right\rangle$ for Upsilon $p_{\mathrm{T}}>0 \mathrm{GeV} / \mathrm{c}$, while both PYTHIA 8 and Herwig with $k_{\perp}>20 \mathrm{GeV} / \mathrm{c}$ show a stronger than linear dependence.

40th International Conference on High Energy physics - ICHEP2020

July 28 - August 6, 2020

Prague, Czech Republic (virtual meeting)

${ }^{*}$ Speaker 


\section{Introduction}

This work focuses on PYTHIA and Herwig simulations of $\Upsilon$ production vs. charged particle multiplicity $\left(N_{\mathrm{ch}}\right)$ in pp collisions at $\sqrt{s}=500 \mathrm{GeV}$. Normalised multiplicity dependence is a meaningful tool for understanding the particle production mechanisms and the interplay between soft and hard QCD processes. Recent experimental results show stronger than linear increase [1, 2]. This may be explained by multi-partion interactions, string percolation or color glass condensate saturation effects.

The experimental observable $N_{\Upsilon} /\left\langle N_{\Upsilon}\right\rangle$ is calculated as $N_{\Upsilon} /\left\langle N_{\Upsilon}\right\rangle=\left(N_{\mathrm{MB}} / N_{\mathrm{MB}}^{\mathrm{bin}}\right)\left(N_{\Upsilon}^{\mathrm{bin}} / N_{\Upsilon}\right)$, where $N_{\mathrm{ch}} /\left\langle N_{\mathrm{ch}}\right\rangle$ is the self-normalised particle multiplicity, $N_{\Upsilon}\left(N_{\mathrm{MB}}\right)$ is the total number of events containing $\Upsilon$ meson (minimum bias events) and $N_{\Upsilon}^{\text {bin }}\left(N_{\mathrm{MB}}^{\mathrm{bin}}\right)$ is the number of $\Upsilon$ (minimum bias) events in the corresponding multiplicity bin.

\section{Simulation}

The simulation consists of two parts: minimum bias and $\Upsilon$ production. For minimum bias events non-single-diffractive soft QCD events were chosen. Criteria for particle selection are: $|\eta|<1, p_{\mathrm{T}}>0.2 \mathrm{GeV} / \mathrm{c}$ (STAR cuts), stable particles $(\tau>10 \mathrm{~mm} / \mathrm{c}$ to reject weak decay contribution). Only directly produced $\Upsilon(1 \mathrm{~S})$ states within $p_{\mathrm{T}}>0$ or $4 \mathrm{GeV} / \mathrm{c}$ in electron decay channel were selected. This study also includes comparison to STAR preliminary data [3].

The Monte Carlo generators used in this study are PYTHIA 8.240 [4] with STAR HF tune and Herwig 7.2.0 [5, 6]. The main features of PYTHIA include $p_{\mathrm{T}}$ ordered showers, Lund string hadronisation model and direct $\Upsilon$ production (dedicated matrix elements for Bottomonia). Herwig's main features are angular ordered showers, cluster hadronisation and $\Upsilon$ production during hadronisation phase ( $b \bar{b}$ matrix element). The results from Herwig depend on b-parton $k_{\perp}$ cut (4 or $20 \mathrm{GeV} / \mathrm{c}$ ) - the value of the $k_{\perp}$ cut influences the $N_{\mathrm{ch}}$ and $p_{\mathrm{T}}$ spectra.

The multiplicity and $p_{\mathrm{T}}$ distributions can be seen in Fig. 1. Both PYTHIA and Herwig minimum bias spectra describe real data reasonably well. For $\Upsilon$ containing events, Herwig with $k_{\perp}>4 \mathrm{GeV} / \mathrm{c}$ is in closest agreement in terms of multiplicity and PYTHIA in terms of $p_{\mathrm{T}}$ distributions [3].
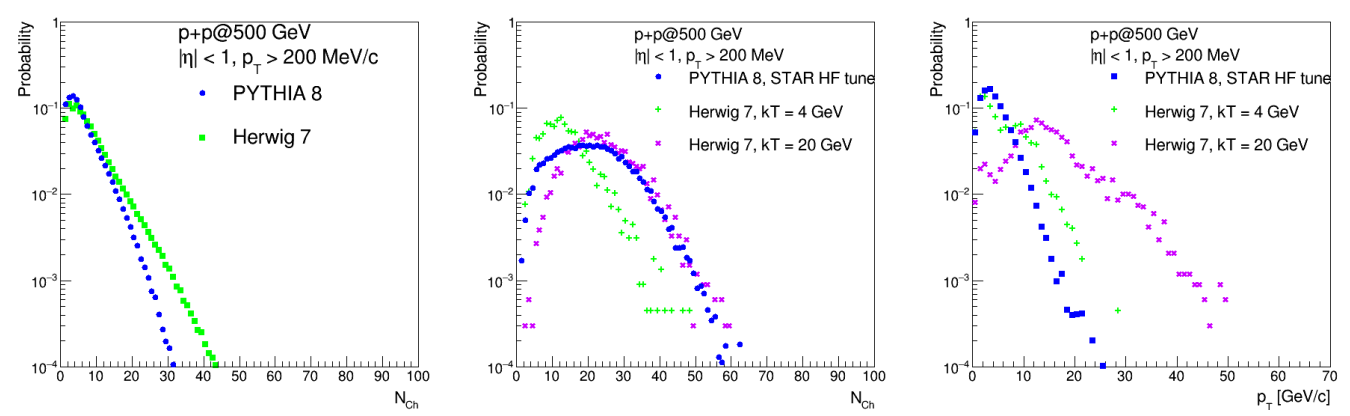

Figure 1: Charged particle multiplicity distributions for MB (left) and $\Upsilon(1 S)$ containing (middle) events and $p_{\mathrm{T}}$ distributions for $\Upsilon$ containing events (right). 


\section{Results and Conclusion}

The results of both Herwig and PYTHIA simulation are compared to STAR preliminary data in Fig. 2.
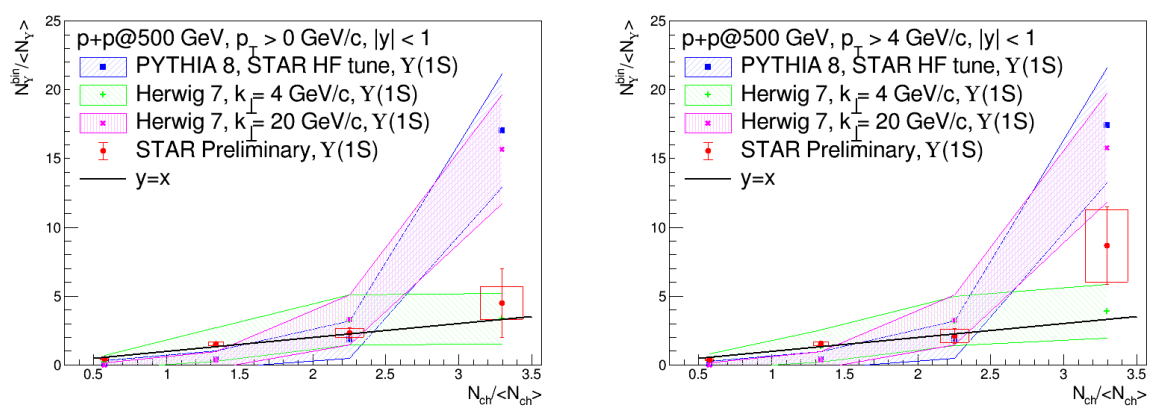

Figure 2: Normalised $\Upsilon(1 \mathrm{~S})$ yield dependence on normalised multiplicity. The PYTHIA simulations for direct $\Upsilon(1 \mathrm{~S})$ and Herwig simulations are compared to STAR preliminary data [3] for $p_{\mathrm{T}}$-integrated (left) and $p_{\mathrm{T}}>4 \mathrm{GeV} / \mathrm{c}$ (right).

STAR $\Upsilon$ data with $p_{\mathrm{T}}>0 \mathrm{GeV} / \mathrm{c}$ are better described by Herwig with $k_{\perp}>4 \mathrm{GeV} / \mathrm{c}$, which is consistent with linear dependence. For $p_{\mathrm{T}}>4 \mathrm{GeV} / \mathrm{c}$, the STAR data indicate a stronger than linear rise, which is qualitatively reproduced by PYTHIA 8 and Herwig with $k_{\perp}>20 \mathrm{GeV} / \mathrm{c}$. It may indicate that Upsilons are produced in multiple parton interactions [2]. However, higher precision measurement as well as dedicated Monte Carlo tunes are needed for a deeper insight.

\section{Acknowledgements}

The work was supported from the project LTT18002 of the Ministry of Education, Youth, and Sport of the Czech Republic and from European Regional Development Fund-Project "Center of Advanced Applied Science" No. CZ.02.1.01/0.0/0.0/16-019/0000778.

\section{References}

[1] J. Adam [STAR], Phys. Lett. B 786 (2018), 87-93

[2] J. Adam et al. [ALICE], JHEP 09 (2015), 148

[3] L. Kosarzewski [STAR], J. Phys.: Conf. Ser. 1667 (2020), 012022

[4] T. Sjöstrand, S. Mrenna and P. Skands, JHEP05 (2006) 026, Comput. Phys. Comm. 178 (2008) 852

[5] M. Bähr et al., Eur. Phys. J. C 58 (2008), 639

[6] J. Bellm et al., Eur. Phys. J. C 76 no.4 (2016), 196 